\title{
Exact Noncommutative Two-Dimensional Hydrogen Atom
}

\author{
B. C. Wang, ${ }^{1}$ E. C. Brenag, ${ }^{1}$ R. G. G. Amorim $\mathbb{D}^{2,3}$ V. C. Rispoli ${ }^{1},{ }^{1}$ and S. C. Ulhoa ${ }^{3,4}$ \\ ${ }^{1}$ Faculdade Gama, Universidade de Brasília, 72444-240 Brasília, DF, Brazil \\ ${ }^{2}$ International Center of Physics and Faculdade Gama, Universidade de Brasília, 72444-240 Brasília, DF, Brazil \\ ${ }^{3}$ Canadian Quantum Research Center, 204-3002 32 Ave Vernon, BC, Canada V1T 2L7 \\ ${ }^{4}$ International Center of Physics, Instituto de Física, Universidade de Brasília, 70910-900 Brasília, Brazil \\ Correspondence should be addressed to R. G. G. Amorim; ronniamorim@gmail.com
}

Received 26 January 2021; Accepted 30 March 2021; Published 8 April 2021

Academic Editor: Sunny Vagnozzi

Copyright (c) 2021 B. C. Wang et al. This is an open access article distributed under the Creative Commons Attribution License, which permits unrestricted use, distribution, and reproduction in any medium, provided the original work is properly cited. The publication of this article was funded by $\mathrm{SCOAP}^{3}$.

\begin{abstract}
In this work, we present an exact analysis of the two-dimensional noncommutative hydrogen atom. In this study, the Levi-Civita transformation was used to perform the solution of the noncommutative Schrodinger equation for Coulomb potential. As an important result, we determine the energy levels for the considered system. Using the result obtained and experimental data, a bound on the noncommutativity parameter was obtained.
\end{abstract}

\section{Introduction}

The concept of noncommutativity in physical theories was formally introduced by Snyder in 1947 [1-3]. In a seminal paper, Snyder stated that spatial coordinates would not commute with each other at small distances. In this sense, a new paradigm was proposed in which the space-time should be understood as a collection of tiny cells of minimum size, where there is no such idea of a point. So far, once the minimum size is reached, in the realm of some high-energy phenomenon, the position should be given by the noncommutative coordinate operators. As a direct consequence, it would be impossible to precisely measure the position of a particle. Over the last years, the interest of the scientific community on noncommutative geometry has increased due to works on nonabelian theories [4], gravitation [5-7], standard model [8-10], and quantum Hall effect [11]. More recently, the discovery that the dynamics of an open string can be associated with noncommutative spaces has contributed to the latest revival of noncommutative theories [12]. Noncommutative physics has a wide range of applications, from noncommutative geometry to corrections in classical systems due to noncommutative coordinates. Particularly, noncommutative geometry has been a promising approach to understanding some limit of quantum gravitation [13]. Some models foresee a possible experimental measurement given the current advance in astrophysical techniques applied to black holes [14].

From the mathematical point of view, the simplest algebra obeyed by the coordinate operators $x \wedge^{\mu}$ is

$$
\left[x \wedge^{\mu}, x \wedge^{\nu}\right]=i \Theta^{\mu \nu}
$$

where $\Theta^{\mu v}$ is a skew-symmetric constant tensor called noncomutativity parameter. It is worth to point out that the mean values of the position operators do correspond to the actual position observed. Thus, it is said that such operators are Hermitian ones. It is well known in quantum mechanics that a noncommutative relation between two operators leads to a specific uncertainty relation; hence, the above expression yields

$$
\Delta x \wedge^{\mu} \Delta x \wedge^{v} \geq \frac{1}{2}\left|\Theta^{\mu v}\right|
$$

which implies a set of uncertainty relations between position coordinates analogous to the Heisenberg uncertainty principle. Following the ideas introduced by Snyder, it is possible to associate the minimum size with a distance of the $\sqrt{\left|\Theta^{\mu v}\right|}$ order of magnitude. Thus, the noncommutative effects turn 
out to be relevant at such scales. Usually, the noncommutativity is introduced by means of the Moyal product defined as [2]

$$
\left.f(x) \star g(x) \equiv \exp \left(\frac{i}{2} \Theta^{\mu \nu} \frac{\partial}{\partial x^{\mu}} \frac{\partial}{\partial y^{\nu}}\right) f(x) g(y)\right|_{y \longrightarrow x},
$$

with a constant $\Theta^{\mu \nu}$. Then, the usual product is replaced by the Moyal product in the classical Lagrangian density. In a similar perspective, the noncommutative quantum mechanics is introduced by imposing further commutation relations between the position coordinates themselves.

In this perspective, we aim to apply the ideas about noncommutativity of space to the hydrogen atom. The hydrogen atom is an electrically neutral atom with a positively charged proton and a negatively charged electron bounded to the nucleus. This system plays a significant role in quantum mechanics and field theory. There are many good reasons to address the hydrogen atom [15]. As an example, the hydrogen atom with high-precision measurements in atomic transitions is one of the best laboratories to test quantum electrodynamics theory [16]. Other applications of the hydrogen atom appear in many occasions, such as to examine the constancy of fine structure constant over a cosmological time scale [17]. There are some approaches to treat the hydrogen atom in a noncommutative space. Such approaches are differentiated by the role of non-commutativity in a specific representation. In this context, it is interesting to note that corrections for Lamb's displacement were obtained in a context of non-commutative quantum electrodynamics [18]. Even non-commutative corrections for the hydrogen atom in a curved space were obtained [19]. In this paper, we analyze the two-dimensional noncommutative hydrogen atom. A two-dimensional hydrogen atom can be defined as a system in which the motion of the electron around the proton is constrained to be planar. Then, in this work, we consider that this plane is noncommutative. As a practical example, a semiconductor quantum well under illumination is a quasi-two-dimensional system, in which photoexcited electrons and holes are essentially confined to a plane $[20,21]$. There are many works that consider the hydrogen atom in a noncommutative context, but they present disagreement in results. Our method presents an approach using the Levi-Civita mapping, which allows an exact treatment.

This paper is organized as follows. In Section 2, we present the mathematical framework of the two-dimensional hydrogen atom. The Levi-Civita mapping is presented in Section 3. In Section 4, we obtain the solution and spectrum for the noncommutative hydrogen atom. Finally, in Section 5, we present our concluding remarks.

\section{Mathematical Framework of Noncommutative Two-Dimensional Hydrogen Atom}

The Hamiltonian that defines two-dimensional hydrogen atom is given by

$$
H=\frac{1}{2 m}\left(p_{x}^{2}+p_{y}^{2}\right)-\frac{k}{\left(x^{2}+y^{2}\right)^{1 / 2}}
$$

where $p_{x}$ and $p_{y}$ stands for the momentum of the electron in directions $x$ and $y$, respectively; $x$ and $y$ represents the electron coordinates and the constant $k=e^{2} / 4 \pi \varepsilon_{o}$, where $e$ is the elementary charge and $\varepsilon_{o}$ is the vacuum electric permittivity. To quantize the Hamiltonian given in Equation (4), as usual, the momentum operators are given by $\widehat{p}_{x}=-i \hbar(\partial /$ $\partial x)$ and $\widehat{p}_{y}=-i \hbar(\partial / \partial y)$, where $\hbar=h / 2 \pi$ and $h$ is the Planck constant.

In the noncommutative perspective, we define the following position operators:

$$
\begin{aligned}
& \hat{x}=x+\frac{i \Theta}{2} \frac{\partial}{\partial y}, \\
& \hat{y}=y-\frac{i \Theta}{2} \frac{\partial}{\partial x},
\end{aligned}
$$

in which $\Theta$ is the noncommutativity parameter in Cartesian coordinates. We notice that

$$
[\widehat{x}, \widehat{y}]=i \Theta
$$

as expected.

However, the treatment of the Hamiltonian given in Equation (4) is difficult because of the operators in the denominator of the potential energy term. For this reason, in the next section, we present a transformation that puts the system in a more suitable way.

\section{Levi-Civita Mapping}

The Levi-Civita (also known as Bohlin) transformation is a parabolic coordinate mapping that is capable of converting the planar Coulomb problem into a two-dimensional harmonic oscillator [22-25]. It is a $\mathbb{R}^{2} \longrightarrow \mathbb{R}^{2}$ surjection defined by

$$
\begin{aligned}
& x=u^{2}-v^{2}, \\
& y=2 u v .
\end{aligned}
$$

Given Equation (7), it is immediate to conclude that

$$
\begin{gathered}
\frac{\partial}{\partial u}=2\left(u \frac{\partial}{\partial x}+v \frac{\partial}{\partial y}\right) \\
\frac{\partial}{\partial v}=2\left(-v \frac{\partial}{\partial x}+u \frac{\partial}{\partial y}\right)
\end{gathered}
$$

and, by inversion,

$$
\frac{\partial}{\partial x}=\frac{1}{2\left(u^{2}+v^{2}\right)}\left(u \frac{\partial}{\partial u}-v \frac{\partial}{\partial v}\right)
$$




$$
\frac{\partial}{\partial y}=\frac{1}{2\left(u^{2}+v^{2}\right)}\left(v \frac{\partial}{\partial u}+u \frac{\partial}{\partial v}\right) .
$$

As a direct consequence of Equation (10), the momentum operators can be rewritten in this new coordinate system as

$$
\begin{aligned}
& p_{x}=\frac{p_{u} u-p_{v} v}{2\left(u^{2}+v^{2}\right)} \\
& p_{y}=\frac{p_{u} v+p_{v} u}{2\left(u^{2}+v^{2}\right)} .
\end{aligned}
$$

It should be noted that the Levi-Civita mapping is a canonical transformation [24].

Applying Equations (7) and (12) in Equation (4), we obtain the following transformed Hamiltonian:

$$
H=\frac{1}{2 m}\left[\frac{p_{u}^{2}+p_{v}^{2}}{4\left(u^{2}+v^{2}\right)}\right]-\frac{k}{\left(u^{2}+v^{2}\right)} .
$$

Finally, the hypersurface defined by $H=E$ is given by

$$
\frac{1}{2 m}\left(p_{u}^{2}+p_{v}^{2}\right)-4 E\left(u^{2}+v^{2}\right)=4 k
$$

Equation (15) is the main result of this section and is the one to be used from now on.

\section{Analysis of Two-Dimensional Hydrogen Atom}

Applying the following set of operators in Equation (15),

$$
\begin{gathered}
\widehat{u}=u+\frac{i \theta}{2} \frac{\partial}{\partial v}, \widehat{v}=v-\frac{i \theta}{2} \frac{\partial}{\partial u}, \\
\widehat{p}_{u}=-i \hbar \frac{\partial}{\partial u}, \widehat{p}_{v}=-i \hbar \frac{\partial}{\partial v},
\end{gathered}
$$

we obtain the modified Schrodinger equation as

$$
\begin{aligned}
& -\left(\frac{\hbar^{2}}{2 m}-E \theta^{2}\right)\left(\frac{\partial^{2} \psi}{\partial u^{2}}+\frac{\partial^{2} \psi}{\partial v^{2}}\right) \\
& -4 E\left[\left(u^{2}+v^{2}\right) \psi-i \theta\left(v \frac{\partial \psi}{\partial u}-u \frac{\partial \psi}{\partial v}\right)\right]=4 k \psi,
\end{aligned}
$$

where $\psi \equiv \psi(u, v)$ is the potential, $\theta$ is the noncommutativity parameter in parabolic coordinates, $[\widehat{u}, \widehat{v}]=i \theta,\left[\widehat{u}, \widehat{p}_{u}\right]=i \hbar$, and $\left[\widehat{v}, \widehat{p}_{v}\right]=i \hbar$. It is crucial to note that the order of $\theta$ is $\theta$ $\sim \sqrt{\Theta}$, due to Equation (6).

The solution of Equation (17) can be obtained from the following change of variables $z=u^{2}+v^{2}$, and then, Equation (12) can be rewritten as

$$
z \frac{d^{2} \psi(z)}{d z^{2}}+\frac{d \psi(z)}{d z}+\frac{1}{\beta}(E z+k) \psi(z)=0,
$$

with $\beta=\left(\left(\hbar^{2} / 2 m\right)-E \theta^{2}\right)$. Defining $\psi(z)=e^{-\lambda z} \phi(z)$, where $\lambda=\sqrt{-E / \beta}$, Equation (18) can be written as

$$
z \frac{d^{2} \phi(z)}{d z^{2}}+(1-2 \lambda z) \frac{d \phi(z)}{d z}+\left(\frac{k}{\beta}-\lambda\right) \phi(z)=0
$$

Performing the change of variable $w=2 \lambda z$, we finally obtain

$$
w \frac{d^{2} \phi(w)}{d w^{2}}+(1-w) \frac{d \phi(w)}{d w}+\frac{1}{2 \lambda}\left(\frac{k}{\beta}-\lambda\right) \phi(w)=0 .
$$

It should be noted that Equation (20) is a special case of Kummer's differential equation [26, 27]. Therefore, its solution can be written in terms of a linear combination of Kummer's confluent hypergeometric functions [26, 27]. However, in this paper, the solution will be written in terms of Laguerre polynomials; that is why we note that Equation (20) has the following form:

$$
w \phi^{\prime \prime}+(1-w) \phi^{\prime}+l \phi=0,
$$

which is the Laguerre differential equation. If $l$ is an integer $l=0,1,2,3, \cdots$, the solution of Laguerre's equation is given by Laguerre polynomials $L_{l}(x)$. It could also be noted that Laguerre polynomials can be defined through confluent hypergeometric functions [26, 27]. Finally, we obtain the solution

$$
\psi(u, v)=e^{-\lambda\left(u^{2}+v^{2}\right)} L_{l}\left(2 \lambda\left(u^{2}+v^{2}\right)\right) .
$$

The energy levels can be determined from

$$
l=\frac{1}{2}\left(\frac{k}{\lambda \beta}-1\right),
$$

with $l=0,1,2,3, \cdots$. Using the condition given in Equation (23), the spectrum can be calculated as

$$
E^{2}-\frac{\hbar^{2}}{2 m \theta^{2}} E-\frac{k^{2}}{n^{2} \theta^{2}}=0
$$

where $n=2 l+1$. Solving Equation (24), we obtain the spectrum of noncommutative two-dimensional hydrogen atom

$$
E_{n}=\frac{\hbar^{2}}{4 m \theta^{2}}-\frac{\hbar^{2}}{4 m \theta^{2}} \sqrt{1+\frac{16 k^{2} m^{2} \theta^{2}}{\hbar^{4} n^{2}}} .
$$

Considering $16 k^{2} m^{2} \theta^{2} / \hbar^{4} n^{2} \ll 1$ and using the binomial series $(1+x)^{j}=1+j x+j(j-1) x^{2} / 2+\cdots$, we calculate the following approximation:

$$
E_{n} \approx \frac{-m e^{4}}{8 \pi^{2} \varepsilon_{o}^{2} \hbar^{2} n^{2}}+\frac{e^{8} m^{3} \theta^{2}}{32 \pi^{4} \varepsilon_{o}^{4} \hbar^{6} n^{4}} .
$$

Notice that in the limit $\theta \longrightarrow 0$, we obtain the same result 
of the usual two-dimensional hydrogen atom given in the literature $[16,17]$. Notice also that the first-order term in $\theta$ does not contribute to the energy of this system.

Then, the noncommutative correction, $\Delta E_{\mathrm{NC}}$, for the energy is given by

$$
\Delta E_{\mathrm{NC}} \approx \frac{e^{8} m^{3} \theta^{2}}{32 \pi^{4} \varepsilon_{o}^{4} \hbar^{6} n^{4}} .
$$

The result given in Equation (27) can be used to estimate the bound on the noncommutativity parameter $\theta$. The experimental value for $1 S \longrightarrow 3 S$ frequency transition in the hydrogen atom is $v_{1 S \longrightarrow 3 S}=(2922743278671.6 \pm 0.9) \mathrm{kHz}$ [28]. The uncertainty in this experimental value $\Delta v=0.9$ $\mathrm{kHz}$ can fix the upper bound on the parameter $\theta$. In this sense, the theoretical value for the error in transition $1 S$ $\longrightarrow 3 S$, denoted by $\Delta E_{1 \longrightarrow 3}$, is given by

$$
\Delta E_{1 \longrightarrow 3} \approx \frac{m^{3} e^{8} \theta^{2}}{32 \pi^{4} \varepsilon_{o}^{4} \hbar^{6}}\left[\frac{80}{81}\right],
$$

Using the fact that in the two-dimensional case, the energy is four times the energy of the three-dimensional case, then $\Delta E_{1 \longrightarrow 3} / 4=h \Delta v$, where $h$ is the Planck constant. So, we have

$$
\theta \lesssim\left[\frac{1296}{5} \frac{\pi^{5} \varepsilon_{o}^{4} \hbar^{7} \Delta v}{m^{3} e^{8}}\right]^{1 / 2}
$$

Performing all the calculations, we obtain $\theta \lesssim 1.4 \cdot 10^{-17}$ $\mathrm{m}$. In this case, the bound of the noncommutativity parameter $\Theta$ is $\Theta \lesssim 1.96 \cdot 10^{-34} \mathrm{~m}^{2}$. Using the definition of the length scale factor, $\Gamma_{\mathrm{NC}}=\sqrt{|\Theta|}$, that is, the length scale where the noncommutative effects of the space will be relevant, we found for the considered case $\Gamma_{\mathrm{NC}} \lesssim 1.4 \cdot 10^{-17} \mathrm{~m}$, which is about one hundred times smaller than the proton radius $r_{p} \approx 0.833 \cdot 10^{-15} \mathrm{~m}$.

It is interesting to note that the noncommutative relationship depends on the coordinates adopted, given that the dimension of the noncommutative parameter itself changes with such a choice. Thus, we denote by $\Theta$ the parameter with dimension of squared distance, which establishes a noncommutative relationship between the Cartesian coordinates. On the other hand, $\theta$ denotes the parameter that establishes this same relationship for parabolic coordinates. There is certainly a nontrivial algebraic relationship between these parameters. But as we are already in an approximate regime, we are guided by dimensional analysis to establish a limit for $\Theta$. Such a limit will be a good approximation for the real order of magnitude of this parameter.

\section{Concluding Remarks}

Using the Levi-Civita mapping, we treated the nontrivial problem of the noncommutative hydrogen atom. As a result, we obtain the solution of the Schrodinger equation for this system and calculate the energy levels. Using the spectrum obtained and experimental data, we estimated the noncommutativity parameter $\Theta$, which has an order of magnitude of $10^{-34} \mathrm{~m}^{2}$, and the noncommutative effects will be relevant to a length smaller than $10^{-17} \mathrm{~m}$. This result has the same order of magnitude obtained in [29], in which the authors studied the hydrogen atom in rotationally invariant noncommutative space. In this way, they founded the corrections to the energy levels of the hydrogen atom up to the second order in the parameter of noncommutativity. The upper bound of the parameter of noncommutativity is estimated on the basis of the experimental results for 1 s to $2 \mathrm{~s}$ transition frequence. The calculations for obtaining the corrected energy levels were performed using a perturbative method while in our research, the calculations of energy levels are performed exactly. Our results are in agreement with the literature [28, 30], bearing in mind that there is no prediction of energy dependence with a noncommutativity parameter linearly. It is interesting to write the parameter of noncommutativity in natural units in order to compare it with possible experimental data. Therefore, it is necessary to divide $\theta$ by $\hbar c$, which results to $\theta=7.11 \cdot 10^{-2} \mathrm{GeV}^{-1}$. It is important to say that the $2 \mathrm{D}$ atom is not a real model; it is more of a toy model that enables us to make some conclusions about the system. It is interesting to notice that the model does not allow to verify a transition between states 1 s and 2 s, because $n$, which appears in the energy, is an odd integer. In the sequence, we intend to analyze the noncommutative three-dimensional hydrogen atom. In addition, in order to obtain better accuracy in estimating of noncommutative parameter, we intend to analyze the noncommutative Zeeman and Stark effects.

\section{Data Availability}

The data used to support the findings of this study are included within the article.

\section{Conflicts of Interest}

The authors declare that they have no conflicts of interest.

\section{Acknowledgments}

This work was partially supported by CAPES and CNPq of Brazil.

\section{References}

[1] R. Jackiw, "Physical instances of noncommuting coordinates," Nuclear Physics B-Proceedings Supplements, vol. 108, pp. 3036, 2002.

[2] H. S. Snyder, "Quantized space-time," Physics Review, vol. 71, no. 1, pp. 38-41, 1947.

[3] H. S. Snyder, "The electromagnetic field in quantized spacetime," Physics Review, vol. 72, no. 1, pp. 68-71, 1947.

[4] A. H. Chanseddine and G. Felder, "Gravity in noncommutative geometry," Communications in Mathematical Physics, vol. 155, no. 1, pp. 205-217, 1993.

[5] W. Kalau and M. Walze, "Gravity, non-commutative geometry and the Wodzicki residue," Journal of Geometry and Physics, vol. 16, p. 327, 1955. 
[6] D. Kastler, "The dirac operator and gravitation," Communications in Mathematical Physics, vol. 166, no. 3, pp. 633-643, 1995.

[7] A. H. Chanseddine and A. Connes, "The spectral action principle," Communications in Mathematical Physics, vol. 186, no. 3, pp. 731-750, 1997.

[8] A. Connes and J. Loot, "Particle models and noncommutative geometry," Nuclear Physics B-Proceedings Supplements, vol. 18B, p. 29, 1991.

[9] J. C. Varilly and J. M. Garcia-Bondia, "Connes' noncommutative differential geometry and the standard model," Journal of Geometry and Physics, vol. 12, no. 4, pp. 223-301, 1993.

[10] C. P. Martin, J. C. Varilly, and J. M. Garcia-Bondia, “The standard model as a noncommutative geometry: the low-energy regime," Physics Reports, vol. 294, p. 363, 1998.

[11] J. Belissard, A. van Elst, and H. Schulz-Baldes, "The noncommutative geometry of the quantum Hall effect," Journal of Mathematical Physics, vol. 35, p. 53, 1994.

[12] N. Seiberg and E. Witten, "String theory and noncommutative geometry," Journal of High Energy Physics, vol. 1999, p. 32, 1999.

[13] H. A. Chamseddine, "Noncommutative gravity," Henri Poincare, vol. 4, no. S2, pp. 881-887, 2003.

[14] A. Ogun, I. Sakalli, J. Saavedra, and C. Leiva, "Shadow cast of noncommutative black holes in Rastall gravity," Modern Physics Letters A, vol. 35, article 2050163, 2020.

[15] D. Palmer, Hydrogen in the Universe, NASA, 2008.

[16] B. Zaslow and M. E. Zandler, "Two-dimensional analog to the hydrogen atom," American Journal of Physics, vol. 35, no. 12, pp. 1118-1119, 1967.

[17] X. L. Yang, S. H. Guo, F. T. Chan, K. W. Wong, and W. Y. Ching, "Analytic solution of a two-dimensional hydrogen atom. I. Nonrelativistic theory," Physical Review A, vol. 43, no. 3, pp. 1186-1196, 1991.

[18] M. Chaichian, M. M. Sheikh-Jabbari, and A. Tureanu, "Hydrogen atom spectrum and the lamb shift in noncommutative QED," Physical Review Letters, vol. 86, no. 13, pp. 27162719, 2001.

[19] V. G. Kupriyanov, "A hydrogen atom on curved noncommutative space," Journal of Physics A: Mathematical and Theoretical, vol. 46, no. 24, p. 245303, 2013.

[20] X. L. Yang, M. Lieber, and F. T. Chan, "The Runge-Lenz vector for the two-dimensional hydrogen atom," American Journal of Physics, vol. 59, no. 3, pp. 231-232, 1991.

[21] M. Chaichian and M. M. Sheik-Jabbari, "Non-commutativity of space-time and the hydrogen atom spectrum," The European Physical Journal C-Particles and Fields, vol. 36, no. 2, pp. 251-252, 2004.

[22] T. Levi-Civita, Opere Mat, vol. 2, p. 417, 1906.

[23] T. Levi-Civita, "Sur la régularisation du problème des trois corps," Acta Mathematica, vol. 42, pp. 99-144, 1920.

[24] P. Campos, M. G. R. Martins, and J. D. M. Viana, "Quantum mechanics on phase space and the Coulomb potential," Physics Letters A, vol. 381, no. 13, pp. 1129-1133, 2017.

[25] M. Kliber and T. Negadi, "On the use of nonbijective canonical transformations in chemical physics," Croatica Chemica Acta, vol. 57, p. 1509, 1984.

[26] A. Erdelyi, W. Magnus, F. Oberhettinger, and F. G. Tricomi, Higher Transcendental Functions, vol. I, McGraw-Hill, 1953.
[27] F. W. J. Olver, D. W. Lozier, R. F. Boisvert, and C. W. Clark, NIST Handbook of Mathematical Functions, Cambridge University Press, 2010.

[28] H. Fleurbaey, S. Galtier, S. Thomas et al., "New measurement of the1S-3S transition frequency of hydrogen: contribution to the proton charge radius puzzle," Physical Review Letters, vol. 120 , no. 18 , p. $183001,2018$.

[29] K. P. Gnatenko and V. M. Tkachuk, "Hydrogen atom in rotationally invariant noncommutative space," Physics Letters A, vol. 378, no. 47, pp. 3509-3515, 2014.

[30] T. W. Hänsch, J. Alnis, P. Fendel et al., "Precision spectroscopy of hydrogen and femtosecond laser frequency combs," Philosophical Transactions of the Royal Society A: Mathematical, Physical and Engineering Sciences, vol. 363, no. 1834, pp. 2155-2163, 2005. 